TITLE:

\title{
Effects of calcaneal eversion on three-dimensional kinematics of the hip, pelvis and thorax in unilateral weight bearing.
}

\section{AUTHOR(S):}

Tateuchi, Hiroshige; Wada, Osamu; Ichihashi, Noriaki

\section{CITATION:}

Tateuchi, Hiroshige ... [et al]. Effects of calcaneal eversion on three-dimensional kinematics of the hip, pelvis and thorax in unilateral weight bearing.. Human movement science 2011, 30(3): 566-573

\section{ISSUE DATE:}

2011-06

URL:

http://hdl.handle.net/2433/141836

\section{RIGHT:}

(C) 2011 Elsevier B.V.; This is not the published version. Please cite only the published version.; この論文は出版社版でありません。引用の際に は出版社版をご確認ご利用ください。 
1 Title Page

2

3 Title of the article:

4 Effects of calcaneal eversion on three-dimensional kinematics of the hip, pelvis, and

$5 \quad$ thorax in unilateral weight bearing

6

7

8 Authors:

9 Hiroshige Tateuchi, PT, MS ${ }^{1}$

10 Osamu Wada, PT, MS ${ }^{2}$

11 Noriaki Ichihashi, PT, PhD ${ }^{1}$

${ }^{1}$ Department of Development and Rehabilitation of Motor Function, Graduate School

14 of Medicine, Kyoto University, Kyoto, Japan

$15{ }^{2}$ Mizuno Orthopaedic Clinic

Correspondence author:

18 Hiroshige Tateuchi, PT, MS

1953 Kawara-cho, Shogoin, Sakyo-ku, Kyoto 606-8507, Japan

20 Phone: +81-75-751-3964

21 Fax: +81-75-751-3909

$22 \quad$ E-mail: tateuchi@hs.med.kyoto-u.ac.jp 


\section{Introduction}

The alignment of the rear foot influences the motion of bones and joints proximal to the foot in the weight-bearing position (Perry, 1983). Foot pronation, which

4 involves eversion of the calcaneus and plantar flexion and adduction of the talus,

5 results in increased medial rotation of the tibia (Rockar, 1995; Perry, 1983), and

6 simultaneously yields medial rotation of the femur (Lafortune et al., 1994). More

7 recently, it was demonstrated that calcaneal eversion influences the alignment of the

8 pelvis (Khamis \& Yizhar, 2007; Pinto et al., 2008). Khamis \& Yizhar (2007) reported a

9 significant increase in anterior pelvic tilt with an induced increase in calcaneal eversion

10 in a bilateral standing position. Further, Pinto et al. (2008) investigated the effects of

11 bilateral and unilateral increases in calcaneal eversion on pelvic alignment in the

12 frontal and sagittal planes during bilateral standing and reported an increase in anterior pelvic tilt with both bilateral and unilateral calcaneal eversion and a lateral

14 pelvic tilt with unilateral calcaneal eversion. demonstrate the effects of calcaneal eversion on pelvic alignment in the kinematic

17 chain. There were, however, some limitations to these studies. One limitation is that

18 the measurements in these studies were obtained under bilateral weight-bearing

19 conditions. Even in the unilateral experiment conducted by Pinto et al. (2008) the 
1 subjects stood on both feet while the unilateral calcaneus was everted. Under bilateral

2 weight-bearing conditions, pelvic alignment could be affected by both leg motions

3 (Botte, 1981; Gurney, 2002). Therefore, the direct effects of calcaneal eversion on

4 three-dimensional pelvic kinematics under unilateral weight-bearing conditions, such

5 as standing on $1 \mathrm{leg}$, are still unknown. The unilateral weight-bearing condition is a

6 common element in many functional daily activities, and the influence of the kinematic

7 chain from the foot may exert a stronger effect on the proximal segment under the

8 unilateral weight-bearing condition than under the bilateral weight-bearing condition.

9 Therefore, it is important to investigate the relationship between calcaneal eversion

10 and the alignment of the proximal segment under unilateral weight-bearing conditions.

The other limitation of previous studies is that the effect of calcaneal eversion on the upper body parts, i.e., up to the thorax, has not been investigated. Excessive

13 pronation has been found to be a causative factor of low back pain (Botte, 1981). It has

14 been speculated that pelvic obliquity because of subtalar joint pronation could induce

15 excessive load on the sacroiliac joint and spinal structures, thereby resulting in low back pain (Botte, 1981; Rothbart \& Estabrook, 1988). However, to the best of our

17 knowledge, no studies have examined the direct relationship between calcaneal eversion and the kinematics of the upper parts of the body. 
1 research findings suggest that plantar pressure distribution or the motion of the

2 calcaneus and tibia as a result of kinematic coupling is different between the low-arch

3 foot and high-arch foot or between a pronated foot and supinated foot (Nawoczenski et

4 al., 1998; Wong et al., 2008). Therefore, variations in foot structure could affect the

$5 \quad$ kinematics of the upper parts of the body under induced calcaneal eversion conditions.

The primary purpose of this study was to investigate the effects of induced

7 calcaneal eversion on the kinematics of the hip, pelvis, and thorax in 3 dimensions.

8 The secondary purpose was to examine whether variations in foot structure affect the

9 kinematic chain in the upper parts of the body under induced calcaneal eversion

10 conditions. We believe that a more complete understanding of the kinematic chain

11 from the foot to the thorax will provide a better basis for assessment of body

12 malalignment.

\section{Methods}

\subsection{Subjects}

Twenty-eight healthy male volunteers [age: $23.4 \pm 2.7$ (mean \pm SD) years, weight: $64.4 \pm 5.5 \mathrm{~kg}$, height: $173.2 \pm 4.1 \mathrm{~cm}$ ] participated in this study. Exclusion criteria included disease of any joint in the lower extremity/spinal joints and

19 neurological disease. Subjects who had feet with rearfoot angles greater than $10.0^{\circ}$ of 
1 eversion or feet with rearfoot angles less than $2.5^{\circ}$ of eversion were excluded based

2 on reference values for healthy subjects established by Cornwall \& McPoil (2004).

3 Measurement of the rearfoot angle is described below. Subjects provided informed

4 consent and the protocol was approved by the Ethics Committee of the Kyoto

$5 \quad$ University Graduate School and Faculty of Medicine.

\subsection{Foot Measurements}

8

To characterize foot structures, the rearfoot angle and arch height were measured. The subjects were asked to stand barefoot with the center of the heels a

10 foot-length apart and toe-out angle of 10 degrees. The rearfoot angle was obtained

11 using a manual goniometer after first marking a line that bisected the subject's

12 posterior calcaneus and lower one-third of the leg. Arch height was measured using a

13 straight ruler as the distance from the inferior aspect of the navicular tuberosity to the

14 floor. Arch height was divided by foot length for standardization. These methods are

15 frequently used in the measurement of foot structures in the standing position

16 (Cornwall \& McPoil, 2004; Donatelli, 1996; Sobel et al., 1999; Williams \& McClay,

17 2000). In the present study, a single experienced physiotherapist performed each of

18 the foot measurements. The intra-rater reliability of the foot measurements was tested

19 for 10 subjects. The foot measurements in the present study showed acceptable 
1 intra-rater reliability with intraclass correlation coefficients $\left(\mathrm{ICC}_{1,1}\right)$ of 0.817 for the

2 rearfoot angle and 0.967 for arch height.

\subsection{Experimental Setup}

Body kinematics were recorded using a 6-camera Vicon motion system (Vicon

6 Nexus; Oxford Metrics Ltd., Oxford, UK) at a sampling rate of $200 \mathrm{~Hz}$. The subjects were clothed in close fitting briefs, and reflective markers were attached to the body according to the Vicon Plug-in-Gait marker placement protocol (full body) by a single

9 investigator. The thoracic segment contained 6 markers: at the $7^{\text {th }}$ cervical and $10^{\text {th }}$

10 thoracic vertebrae, jugular notch, xiphoid process of the sternum, and left and right

11 acromioclavicular joints. The pelvic segment had 4 markers: at the left and right

12 anterior superior iliac spine and left and right posterior superior iliac spine. Two markers were applied along the line vertically bisecting the calcaneus. All data were

14 low-pass filtered using a Woltring filter with a cut-off frequency of $6 \mathrm{~Hz}$.

\subsection{Protocol}

Prior to data collection for unilateral standing, data were collected for $10 \mathrm{~s}$ in the bilateral standing position, which was the same position described previously for foot measurements. The bilateral standing position was used as a reference for calculating 
1 the angle in the unilateral standing posture. Trials under 3 unilateral standing

2 conditions were then conducted. The 3 conditions included normal (E0; standing

3 directly on the floor), a $5^{\circ}$ calcaneal eversion condition (E5), and a $10^{\circ}$ calcaneal

4 eversion condition (E10). Altered calcaneal eversion was achieved by placing a

5 medially tilted wooden wedge, which was wider than the plantar surface, covered with

6 a nonslip mat at $5^{\circ}$ and $10^{\circ}$ under the right leg. Subjects were asked to stand on 1 leg

7 with a toe-out angle of $10^{\circ}$ and to flex the contralateral hip to $30^{\circ}$ while maintaining

8 neutral abduction/adduction and rotation, with their hands on their abdomen (Fig. 1).

9 Before experimental data collection, the subjects practiced standing on 1 leg under

10 each condition several times to become familiar with the task. The subjects were then

11 instructed to stand on 1 leg, while looking straight ahead for $10 \mathrm{~s}$. The eversion conditions were measured in a random order. Measurements were repeated 3 times

13 for each task.

\subsection{Data Analysis}

Kinematic parameters measured included the angle of the thorax, pelvis, hip

17 joint, and calcaneal eversion. The angle of the thorax and the pelvic segment were measured with reference to the global frame. The hip angle was defined as the relative

19 angle of the thigh axis, defined as the center of the knee joint to the center of the hip 
1 joint, to the pelvis in the local frame. The angles of the thorax, pelvis, and hip joint were

2 calculated in three dimensions. The calcaneal eversion angle was calculated in the

3 frontal plane based on the method used by Khamis \& Yizhar (2007), and was defined

4 as the relative angle between the tibial axis, defined as a line connecting the proximal

5 and distal centers of the tibia, and a line formed by two markers bisecting the heel. All

6 parameters were calculated with reference to the initial bilateral standing position.

Although recording was carried out over $10 \mathrm{~s}$ for each task, a latter $5 \mathrm{~s}$ was

8 adopted to the analysis to eliminate the initial unstable phase associated with the

9 transition from the initial stance to standing on 1 leg. The mean value over this $5 \mathrm{~s}$ was

10 calculated for all parameters, and the mean value of 3 trials was determined for each

11 subject.

\subsection{Statistical Analysis}

For each parameter, a paired t-test with Bonferroni adjustment was used to determine whether differences existed among the 3 conditions. A $P$ value of $<0.016$ was considered significant for the paired t-test. Pearson product moment correlation coefficients ( $r$ ) were calculated to examine the relationships between foot measurements and changes in kinematics of the thorax, pelvis, and hip joint from the

19 normal condition to $10^{\circ}$ eversion. A $P$ value of $<0.05$ was considered significant for the 

4 USA). at E0.

correlation analysis. ICC $\mathrm{I}_{1,3}$ was calculated to determine the measurement consistency for each variable under calcaneal eversion conditions. All statistical procedures were performed using the SPSS 17.0 statistical analysis package (SPSS Inc., Chicago, IL,

\section{Results}

Acceptable $\mathrm{ICC}_{1,3}$ values, those between 0.878 and 0.992 , were recorded for all kinematics parameters under calcaneal eversion conditions.

Significant differences in calcaneal eversion were observed among the 3 conditions (Fig. 2). Descriptive statistics for kinematics of the hip joint, pelvis, and thoracic segment are shown in Table 1. The hip flexion angle significantly increased with an increase in induced calcaneal eversion. The hip adduction angle significantly increased at E5 compared with the E0; however, a decreased hip adduction angle was found at E10 compared with E5, and no significant difference was observed between E0 and E10. The hip medial rotation angle was significantly larger at E5 and E10 than

The pelvic anterior tilt displayed a steady increase with an increase in calcaneal eversion, although there was no significant difference between E0 and E5. The pelvic lateral tilt to standing side was significantly smaller at E5 than at E0 and E10. There 
were no significant differences among the 3 conditions for pelvic axial rotation.

In the sagittal plane, there were no significant differences in thoracic kinematics.

Thoracic lateral tilt and axial rotation to standing side were significantly increased at E5 and E10 compared with E0.

The relationships between the foot measurements and changes in kinematics from E0 to E10 are shown in Table 2. Arch height was significantly negatively correlated with thoracic axial rotation to standing side $(r=-0.528, P<0.01)$.

\section{Discussion}

The present study demonstrates a transferred effect of calcaneal eversion up to the thorax in 3 dimensions under unilateral weight-bearing conditions.

The measured calcaneal eversion angle consistently increased by approximately $1.5^{\circ}$ with each change in test conditions. This result suggests the validity of use of the wooden wedge as a tool to induce calcaneal eversion. Analogous to the results of Khamis \& Yizhar (2007). the results of the present study demonstrated that compared with normal condition, calcaneal eversion induced hip medial rotation.

This finding may account for the high incidence of hip injury, such as iliotibial band friction syndrome, seen in people with excessive pronation (Hintermann \& Nigg, 1998). Anterior pelvic tilt tended to increase under eversion conditions. This result is 

consistent with Khamis \& Yizhar (2007) and Pinto et al.(2008). However, the mean values for pelvic tilt indicate that the change in pelvic tilt in the sagittal plane occurred within a more posteriorly tilted position of the pelvis than in the bilateral standing position. It has been found that unilateral hip flexion is accompanied by a pelvic posterior tilt in standing (Murray et al., 2002). Thus, it may be that, although there was a statistically significant increase in tilting of the pelvis toward the anterior, calcaneal eversion while standing on 1 leg cannot induce a larger anterior tilt of the pelvis than in bilateral standing.

Interestingly, in the frontal plane, the pelvic lateral tilt to standing side decreased at $5^{\circ}$ eversion compared to the normal; conversely, the pelvic lateral tilt to standing side increased at $10^{\circ}$ eversion compared with $5^{\circ}$ eversion. Hip medial rotation was increased at $5^{\circ}$ eversion compared to normal. Hip abduction, in addition to medial rotation, coils and tightens all of the hip ligaments and capsule (Neumann, 2002). Therefore, it is assumed that the hip joint positioned in medial rotation generates hip adduction motion to moderate the tension of the hip ligaments at $5^{\circ}$ calcaneal eversion. At $10^{\circ}$ eversion, however, the pelvis tilted conversely toward standing side. The increased calcaneal eversion of approximately $2.8^{\circ}$ at $10^{\circ}$ eversion in the present study is larger than that of the approximately $1.4^{\circ}$ eversion observed in a previous study of walking (Cornwall \& McPoil, 2004). This suggests that the $10^{\circ}$ eversion task in 
1 the present study may have produced excessive calcaneal eversion beyond the

2 changes normally experienced during daily activities. The excessively pronated foot

3 tends to load more medially (Willems et al., 2006; Wong et al., 2008). Thus, at $10^{\circ}$

4 eversion, subjects may have had to tilt the pelvis toward standing side in order to

5 recover their postural balance. Clinically, these findings may be significant, calcaneal

6 malalignment might be one of the cause of the pelvic tilting especially in sagittal and

7 frontal plane.

In the frontal plane, thoracic segment tilted toward the standing side under

9 calcaneal eversion. In particular under the $5^{\circ}$ eversion condition, lateral tilt of the

10 thorax to the standing side was higher than under the normal condition, despite the

11 fact that the pelvic tilt toward the standing side was less than that under the normal condition. This finding suggests that lateral tilt of spinal segments between the thorax and pelvis was induced by calcaneal eversion. Axial rotation of the thoracic segment toward the standing side significantly increased under the calcaneal eversion condition.

15 However, pelvic axial rotation did not changed with calcaneal eversion; this indicates that increased rotation of the thorax may contribute to a shift in upper body mass

17 toward the standing side combined with a lateral tilt of the pelvis, rather than contribute

18 to controlling the rotation momentum induced by calcaneal eversion. The finding of

19 increased thoracic rotation without pelvic rotation suggests that rotation of the spine 
1 between the thorax and pelvis was also increased due to calcaneal eversion. Frequent

2 rotation of the spine has been identified as a risk factor for low back pain (Duncan \&

3 Ahmed, 1991; Gordon et al., 1991; Manning et al., 1984; Marras \& Granata, 1995;

4 Schmidt et al., 2007). However, the motion of the each spinal segment was not

5 evaluated directly in this study; further studies will be necessary to examine the

6 influence of calcaneal alignment on spinal malalignment.

We found that the lower the arch height, the larger the thoracic axial rotation

8 toward standing side. A low-arch foot has a larger contact area on the medial aspect of

9 its plantar surface; therefore, it may be accompanied by load shifting in the medial

10 direction (Wong et al, 2008). This medial shift in loading could be compensated for by

11 a shift of the upper body toward standing side to maintain postural balance; however,

12 in the present study, the thoracolumbar spine was bended toward the raised-leg side in

13 response to pelvic lateral tilt. Consequently, it is likely that the thoracic segment was

14 rotated to shift the mass of the upper body to standing side. This finding may have

15 implications for clinicians who assess postural malalignment in patients with low-arch

16 feet. However, this study revealed the immediate effects of calcaneal eversion, and

17 patients with foot pathologies might alter their posture to adapt to the underlying

18 deformity of the foot. Further study on patients with foot pathologies may provide a

19 better understanding of the kinematic chain from the foot to the hip, pelvis, and thorax. 


\section{Conclusions}

In conclusion, we found that wedge induced eversion conditions affect the three

4 dimensional kinematics of hip, pelvis, and thorax except for pelvic axial rotation. In

5 addition, arch height was negatively correlated with change in thoracic axial rotation to

6 standing side from the normal to $10^{\circ}$ eversion condition. The findings of the present

7 study highlight the need for clinicians to consider foot alignment when examining

8 patients with malalignment, such as hip medial rotation, pelvic tilt, and thoracic axial

9 rotation.

\section{Conflict of interest statement}

12 None of the authors have any conflicts of interest associated with this study.

\section{References}

15 Botte, R. R. (1981). An interpretation of the pronation syndrome and foot types of patients with low back pain. Journal of the American Podiatric Medical Association, 71, 243-253. 
1 Donatelli, R. A. (1996). The biomechanics of the foot and ankle. (2nd ed.). Philadelphia: FA Davis.

Duncan, N.A., \& Ahmed, A.M. (1991). The role of axial rotation in the etiology of unilateral disc prolapsed. An experimental and finite-element analysis. Spine, 16, 1089-1098.

Gurney, B. (2002). Leg length discrepancy. Gait \& Posture, 15, 195-206.

Khamis, S., \& Yizhar, Z. (2007). Effect of feet hyperpronation on pelvic alignment in a standing position. Gait \& Posture, 25, 127-134.

Hintermann, B., \& Nigg, B. M. (1998). Pronation in runners. Implications for injuries. Sports Medicine, 26, 169-176.

Lafortune, M. A., Cavanagh, P. R., Sommer, H. J., \& Kalenak, A. (1994). Foot inversion-eversion and knee kinematics during walking. Journal of Orthopaedic Research, 12, 412-420.

Manning, D. P., Mitchell, R. G., \& Blanchfield, L. P. (1984). Body movements and events contributing to accidental and nonaccidental back injuries. Spine, 9, 734-739.

Marras, W.S., \& Granata, K. P. (1995). A biomechanical assessment and model of axial twisting in the thoracolumbar spine. Spine, 20, 1440-1451.

Murray, R., Bohannon, R., Tiberio, D., Dewberry, M., \& Zannotti, C. (2002). 
Pelvifemoral rhythm during unilateral hip flexion in standing. Clinical Biomechanics, 17, 147-151.

Nawoczenski, D. A., Saltzman, C. L., \& Cook, T. M. (1998). The effect of foot structure on the three-dimensional kinematic coupling behavior of the leg and rear foot. Physical Therapy, 78, 404-416.

Neumann, D. A. (2002). Kinesiology of the musculoskeletal system. Foundations for physical rehabilitation. St. Louis: Mosby.

Perry, J. (1983). Anatomy and biomechanics of the hindfoot. Clinical Orthopaedics, 177, 9-15.

Pinto, R. Z. A., Souza, T. R., Trede, R. G., Kirkwood, R. N., Figueiredo, E. M., \& Fonseca, S. T. (2008). Bilateral and unilateral increase in calcaneal eversion affect pelvic alignment in standing position. Manual Therapy, 13, 513-519.

Rockar, P. A. (1995). The subtalar joint: Anatomy and joint motion. The Journal of Orthopaedic and Sports Physical Therapy, 21, 361-372.

Rothbart, B. A., \& Estabrook, L. (1988). Excessive pronation: A major biomechanical determinant in the development of chondromalacia and pelvic lists. The Journal of Manual \& Manipulative Therapy, 11, 373-379.

Schmidt, H., Kettler, A., Rohlmann, A., Claes, L., \& Wilke, H. J. (2007). The risk of disc prolapses with complex loading in different degrees of disc degeneration. A finite 
element analysis. Clinical Biomechanics , 22, 988-998.

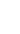

Sobel, E., Levitz, S. J., Caselli, M. A., Tran, M., Lepore, F., Lilja, E., Sinaie, M., \& Wain, E. (1999). Reevaluation of the relaxed calcaneal stance position. Reliability and normal values in children and adults. Journal of the American Podiatric Medical Association, 89, 258-64.

Willems, T. M., Clercq, D.D., Delbaere, K., Vanderstraeten, G., Cock, A.D., \& Witvrouw, E. (2006). A prospective study of gait related risk factors for exercise-related lower leg pain. Gait \& Posture, 23, 91-98.

Williams, D. S., \& McClay, I. S. (2000). Measurements used to characterize the foot and the medial longitudinal arch: Reliability and validity. Physical Therapy, 80, 864-871.

Wong, L., Hunt, A., Burns, J., \& Crosbie, J. (2008). Effect of foot morphology on center-of-pressure excursion during barefoot walking. Journal of the American Podiatric Medical Association, 98, 112-117. 
Fig 1. Measuring position in $5^{\circ}$ eversion condition with applied marker set.

Fig 2. Comparison of the calcaneal eversion angle between three conditions. Normal condition: $2.01 \pm 1.52^{\circ}, 5^{\circ}$ eversion condition: $3.50 \pm 1.66^{\circ}, 10^{\circ}$ eversion condition: $4.83 \pm 2.15^{\circ}$. $\dagger$ Significant difference to value in normal condition. $\ddagger$ Significant difference to value in $5^{\circ}$ eversion condition. 


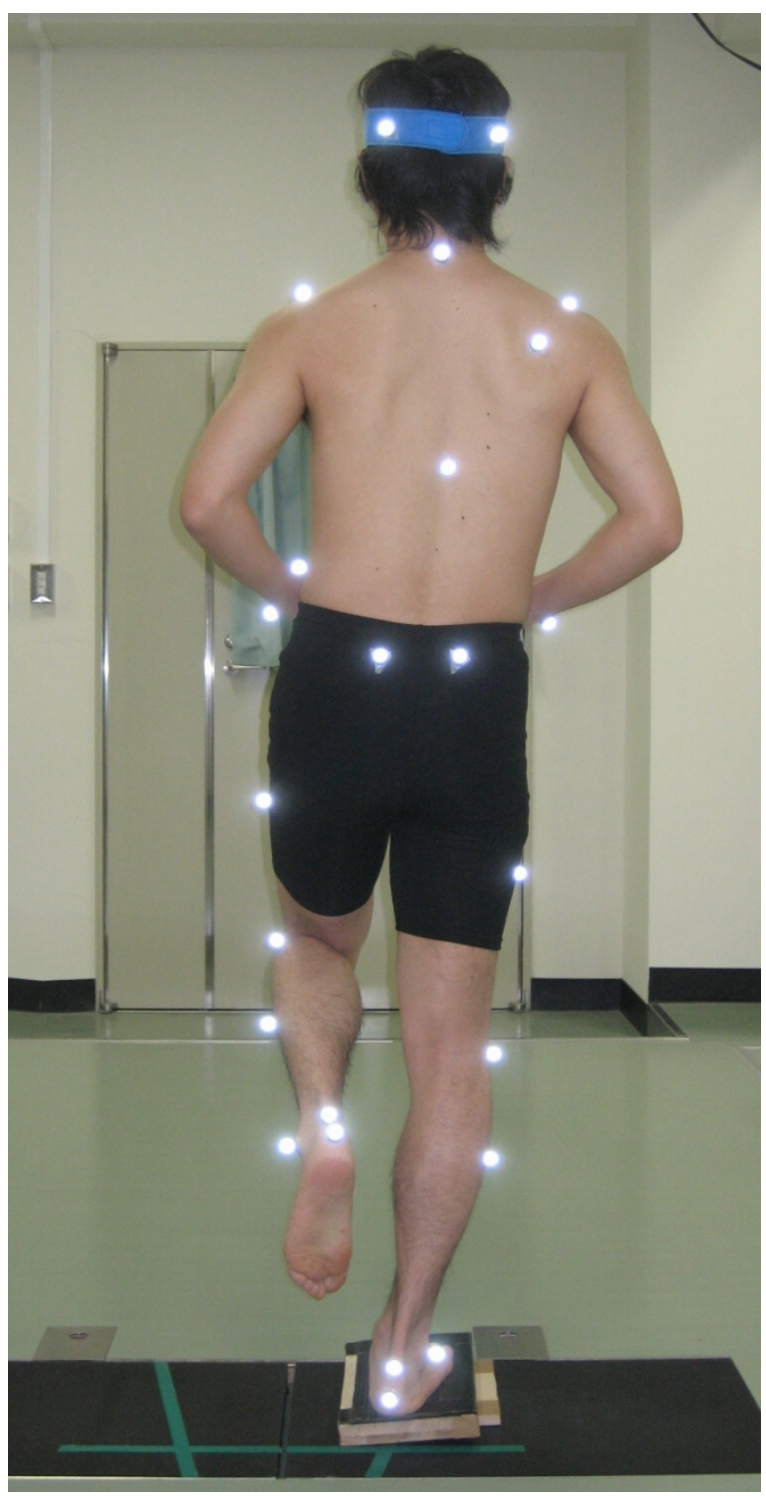

Fig 1. 


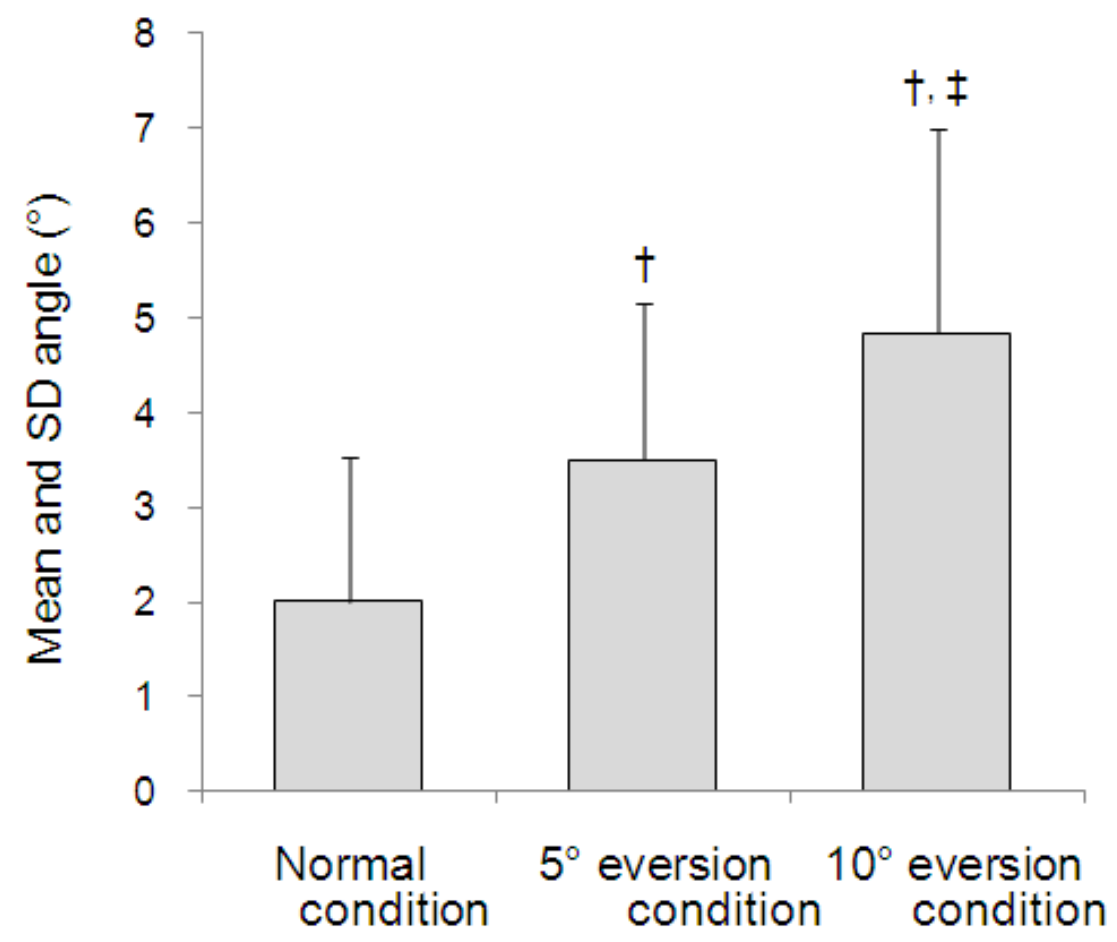

Fig. 2 


\section{Tables}

Table 1. Effects of calcaneal eversion on kinematics of the hip joint, and pelvic and thoracic segment in three dimensions

\begin{tabular}{|c|c|c|c|c|c|c|}
\hline \multirow[b]{2}{*}{ Hip joint $\left(^{\circ}\right)$} & \multirow[b]{2}{*}{ Sagittal (Flexion/Extension) } & \multirow{2}{*}{$\begin{array}{c}\begin{array}{c}\text { Normal } \\
\text { condition }\end{array} \\
(\text { Mean } \pm \text { SD) }\end{array}$} & \multirow{2}{*}{$\begin{array}{r}\begin{array}{c}5^{\circ} \text { eversion } \\
\text { condition }\end{array} \\
(\text { Mean } \pm \mathrm{SD})\end{array}$} & \multicolumn{3}{|c|}{$\begin{array}{c}10^{\circ} \text { eversion } \\
\text { condition } \\
(\text { Mean } \pm \text { SD })\end{array}$} \\
\hline & & & & $\dagger$ & $1.71 \pm 3.41$ & $\dagger, \ddagger$ \\
\hline & Frontal (Adduction/Abduction) & $3.01 \pm 4.05$ & $4.37 \pm 4.19$ & $\dagger$ & $3.19 \pm 4.87$ & $\ddagger$ \\
\hline & Transverse (Medial rotation/Lateral rotation) & $2.15 \pm 3.83$ & $9.52 \pm 13.24$ & $\dagger$ & $9.52 \pm 13.32$ & $\dagger$ \\
\hline \multirow[t]{3}{*}{ Pelvic segment $\left({ }^{\circ}\right)$} & Sagittal (Anterior tilt/Posterior tilt) & $-4.09 \pm 2.56$ & $-3.23 \pm 3.10$ & & $-2.52 \pm 3.13$ & $\dagger, \ddagger$ \\
\hline & Frontal (Lateral tilt) & $6.51 \pm 2.14$ & $5.37 \pm 3.04$ & $\dagger$ & $6.77 \pm 3.54$ & $\ddagger$ \\
\hline & Transverse (Axial rotation) & $-0.50 \pm 2.97$ & $-0.03 \pm 4.63$ & & $-0.91 \pm 4.20$ & \\
\hline \multirow[t]{3}{*}{ Thoracic segment $\left({ }^{\circ}\right)$} & Sagittal (Anterior tilt/Posterior tilt) & $0.10 \pm 1.73$ & $0.02 \pm 2.73$ & & $0.10 \pm 3.14$ & \\
\hline & Frontal (Lateral tilt) & $2.55 \pm 1.50$ & $4.09 \pm 3.02$ & $\dagger$ & $3.98 \pm 3.72$ & $\dagger$ \\
\hline & Transverse (Axial rotation) & $-0.40 \pm 2.31$ & $1.34 \pm 3.51$ & $\dagger$ & $1.25 \pm 3.52$ & $\dagger$ \\
\hline
\end{tabular}

Positive values are flexion, adduction, and medial rotation for hip joint, anterior tilt, and lateral tilt and axial rotation toward the standing side for pelvic and thoracic segment.

† Significant difference to value in normal condition.

‡ Significant difference to value in $5^{\circ}$ eversion condition.

Bonferroni-adjusted $\mathrm{p}$-value was set at 0.016 . 
Table 2. Correlations ( $r$ ) between foot measurements and changes of the kinematics from normal condition to $10^{\circ}$ eversion condition

\begin{tabular}{llcc}
\hline & & Rear foot angle & Arch height \\
\hline Hip joint & Sagittal (Flexion/Extension) & 0.191 & -0.261 \\
& Frontal (Adduction/Abduction) & 0.225 & 0.056 \\
& Transverse (Medial rotation/Lateral rotation) & -0.196 & 0.001 \\
Pelvic segment & Sagittal (Anterior tilt/Posterior tilt) & 0.004 & -0.088 \\
& Frontal (Lateral tilt) & -0.190 & -0.063 \\
& Transverse (Axial rotation) & 0.170 & -0.290 \\
Thoracic segment & Sagittal (Anterior tilt/Posterior tilt) & 0.051 & -0.147 \\
& Frontal (Lateral tilt) & 0.234 & -0.341 \\
& Transverse (Axial rotation) & 0.240 & $-0.528^{\star}$ \\
\hline
\end{tabular}

Positive value for rear foot angle is calcaneal eversion.

* Significant correlation at $P<0.01$. 